\title{
MODULAR REPRESENTATION ALGEBRAS ${ }^{1}$
}

\author{
BY T. A. HANNULA, T. G. RALLEY AND I. REINER
}

Communicated by I. Reiner, July 25, 1966

Let $G$ be a cyclic $p$-group, $K$ a field of characteristic $p$, and $K G$ the group algebra of $G$ over $K$. The representation ring $a(K G)$ is generated by symbols $[M]$, one for each isomorphism class $\{M\}$ of finitely generated left $K G$-modules, with relations

$$
[M]+\left[M^{\prime}\right]=\left[M \oplus M^{\prime}\right],[M][N]=\left[M \otimes_{K} N\right] .
$$

The representation algebra $A(K G)$ is defined as $C \otimes_{z} a(K G)$, where $Z$ is the ring of rational integers, $C$ the complex field. The aim of this note is to give a simple proof of the following theorem of Green [1].

THEOREM. The representation algebra $A(K G)$ is semisimple.

Since $G$ is a cyclic $p$-group, the algebra $A(K G)$ is finite dimensional (and commutative), having $C$-basis $\left\{v_{1}, \cdots, v_{q}\right\}$, where $q=[G: 1]$, and where $v_{r}=\left[V_{r}\right]$. Here, $V_{r}$ denotes the unique indecomposable $K G$-module of dimension $r$. We set $A_{0}=R \otimes_{z} a(K G)$, where $R$ is the real field. Then $A(K G)=C \otimes_{R} A_{0}$, and it suffices to prove that $A_{0}$ is semisimple, or equivalently, that $A_{0}$ has no nonzero elements of square zero.

By the components of a module we mean the indecomposable summands in a direct sum decomposition of the module.

Lemma 1 (Roth [4], Ralley [3]). The number of components of $V_{r} \otimes V_{s}$ is precisely $\min (r, s)$.

PROOF. Let $H_{r}$ be the $r \times r$ matrix with 1's above the main diagonal and zeros elsewhere, let $E_{r}$ be the $r \times r$ identity matrix, and let $\lambda$ be an indeterminate over $K$. Then the number of components of $V_{r} \otimes V_{s}$ is the same as the number of invariant factors of $\left(\lambda E_{r}+H_{r}\right)^{8}$ different from 1 . This easily yields the desired result.

Let us write

$$
v_{r} v_{s}=\sum_{t=1}^{q} a_{r s t} v_{t}, \quad 1 \leqq r, s \leqq q .
$$

Then the coefficients $\left\{a_{r s t}\right\}$ are nonnegative integers, and Lemma 1 asserts that

1 This research was supported by the National Science Foundation. 


$$
\sum_{t=1}^{q} a_{r a t}=\min (r, s), \quad 1 \leqq r, s \leqq q .
$$

Lemma 2. The quadratic form

$$
\sum_{r, s=1}^{q} \min (r, s) X_{r} X_{\varepsilon}
$$

is positive definite.

Proof. One verifies that the given form coincides with $\left(X_{1}+\cdots+X_{q}\right)^{2}+\left(X_{2}+\cdots+X_{q}\right)^{2}+\cdots+X_{q}^{2}$.

We now show that if $u \in A_{0}$ satisfies $u^{2}=0$, then necessarily $u=0$. Write $u=\sum_{r=1}^{a} \alpha_{r} v_{r}, \alpha_{r} \in R$. Then

$$
0=u^{2}=\sum_{r, s} \alpha_{r} \alpha_{s} v_{r} v_{s}=\sum_{r, s, t} \alpha_{r} \alpha_{s} a_{r s t} v_{t},
$$

whence

$$
\sum_{r, s} \alpha_{r} \alpha_{s} a_{r s t}=0, \quad 1 \leqq t \leqq q .
$$

Summing on $t$, we obtain

$$
\sum_{r, s} \min (r, s) \alpha_{r} \alpha_{i}=0,
$$

so by Lemma $2, \alpha_{r}=0$ for $1 \leqq r \leqq q$. This completes the proof.

The above technique has also been used by Hannula [2].

\section{REFERENCES}

1. J. A. Green, The modular representation algebra of a finite group, Illinois J. Math. 6 (1962), 607-619.

2. T. A. Hannula, Group representations over integers modulo a prime power, Ph.D. Thesis, University of Illinois, Urbana, Ill., 1967.

3. T. G. Ralley, Decompositions of products in modular representation rings, Ph.D. Thesis, University of Illinois, Urbana, Ill., 1967.

4. W. E. Roth, On direct product matrices, Bull. Amer. Math. Soc. 40 (1934), 461-468.

UNIVERSITY OF ILLINOIS 